\title{
Analogy instructions promote efficiency of cognitive processes during hockey push-
}

\section{pass performance}

\author{
Tina van Duijn ${ }^{\mathrm{a} *}$
}

Merel C. J. Hoskens ${ }^{\mathrm{a}}$

Rich S. W. Masters ${ }^{\mathrm{a}, \mathrm{c}}$

${ }^{a}$ Te Huataki Waiora Faculty of Health, Sport and Human Performance, University of Waikato, Hamilton, New Zealand

c School of Public Health, Li Ka Shing Faculty of Medicine, University of Hong Kong Hong Kong SAR, China

\author{
*Corresponding author: \\ Tina van Duijn \\ Te Huataki Waiora Faculty of Health, Sport and Human Performance \\ University of Waikato \\ Hamilton 3216, New Zealand \\ Tel \# +64 78384466 ext. 6530 / E-mail address: tv24@students.waikato.ac.nz
}

Preprint version, 8/10/18, ¿American Psychological Association, 2018. This paper is not the copy of record and may not exactly replicate the authoritative document published in the APA journal. Please do not copy or cite without author's permission. The final article is available, upon publication, at: http://dx.doi.org/10.1037/spy0000142 


\title{
Analogy instructions promote efficiency of cognitive processes during hockey push-pass performance
}

\begin{abstract}
Analogy instructions may promote effective skill acquisition by providing movement specific information that can be processed as a single, meaningful unit, rather than as separate 'bits' of information (Liao \& Masters, 2001; Masters, 2000). Behavioural evidence suggests that information processing associated with analogy instructions is less effortful than information processing associated with explicit instructions, resulting in reduced verbal-analytical involvement in movement control (Lam, Maxwell, \& Masters, 2009b).
\end{abstract}

This experiment was designed to test whether analogy instructions promote higher psychomotor efficiency, characterised by greater high-alpha power in the left hemisphere of the brain (Hillman, Apparies, Janelle, \& Hatfield, 2000) and reduced co-activation between the verbal processing (left temporal lobe T7) and motor planning regions of the brain (frontal midline Fz, Hatfield \& Hillman, 2001) during motor performance.

Novices practiced a hockey push-pass task using an analogy instruction, explicit instructions or no instructions (control). Push-pass accuracy during a combined task (passing coupled with decisionmaking) was significantly better following the analogy instruction, which suggested that information processing was less effortful. Left-temporal (T7) EEG high-alpha power was significantly higher in the analogy condition, but T7-Fz co-activation was not significantly different among the conditions.

It is possible that the analogy instruction influenced verbal aspects of information processing without impacting on efficiency of motor planning. Consequently, an analogy instruction may promote cognitive, rather than psychomotor, efficiency.

Keywords: Analogy motor learning, EEG alpha power, EEG coherence, psychomotor efficiency 
Implicit motor learning research has shown that during early stages of learning it can be advantageous to accrue only a minimum of declarative information about the movement to be learned (Masters, 1992). Various forms of implicit motor learning have been developed, including error-reduced learning (e.g., Maxwell, Masters, Kerr, \& Weedon, 2001), dual-task learning (e.g., Maxwell, Masters, \& Eves, 2000) and analogy learning (e.g., Liao \& Masters, 2001). Benefits have been reported during performance under pressure (Hardy, Mullen, \& Jones, 1996; Koedijker, Oudejans, \& Beek, 2007; Lam, Maxwell, \& Masters, 2009a, 2009b; Liao \& Masters, 2001; Masters, 1992), fatigue (Masters, Poolton, \& Maxwell, 2008; Poolton, Masters, \& Maxwell, 2007) and when decisions have to be made during performance (Masters, Poolton, Maxwell, \& Raab, 2008; Poolton, Masters, \& Maxwell, 2006). Performance of implicitly learned tasks has also been shown to remain stable when a cognitively challenging second task is completed concurrently (Koedijker et al., 2011, 2007; Lam et al., 2009b; Masters, Lo, Maxwell, \& Patil, 2008; Maxwell et al., 2001; Poolton et al., 2006).

Analogy learning is a form of motor learning in which an analogical instruction is presented instead of declarative rules about the movement. An analogy conveys information about a complex construct or task (in this case, a motor task) by way of comparison with a simple, familiar concept, often in the form of an image. Liao and Masters (2001) provided empirical evidence that analogy learning benefits performance similarly to other methods of implicit motor learning (e.g., errorless learning, secondary task learning). Table tennis novices were instructed that in order to learn a topspin forehand they should "strike the ball while bringing the bat up the hypotenuse of [a] triangle” (Liao \& Masters, 2001, p. 310). No other information was provided. Performance of participants instructed by analogy was robust both in a dual-task situation and under psychological stress, compared to participants instructed explicitly with rules for how to hit a topspin forehand. Other research has since confirmed that when a skill is learned by analogy instead of declarative rules, performance remains stable under pressure (e.g., in an adapted basketball task, Lam, Maxwell, \& Masters, 2009), when high-complexity decisions have to be made concurrently (Masters, Poolton, Maxwell, et al., 2008; Poolton et al., 2006), and in dual-task settings (Lam et al., 2009b). 
The exact mechanism by which analogy instructions influence motor learning and performance remains ambiguous. Donnelly and McDaniel (1993) showed that analogy learning leads to accrual of less verbalisable (but more inferential) knowledge about scientific concepts, which is one of the defining characteristics of implicit learning (Berry \& Broadbent, 1984; Hayes \& Broadbent, 1988). Liao and Masters (2001) found that analogy learners accrued only a small amount of explicit knowledge about the motor task that they were learning. Similarly, Schücker, Ebbing, and Hagemann (2010) showed that analogy learning was associated with less awareness of skill-related factors under pressure compared to explicit learning. Worry (under pressure) or a cognitive secondary task can disrupt motor planning and performance if the limited capacity of working memory, the mental domain for short-term storage and information processing, is reduced by a cognitive load. However, experimental studies suggest that motor skills that are acquired implicitly do not rely on working memory to the same extent as skills acquired explicitly; processing of these skills is more efficient and performance is less likely to be disrupted (e.g., Maxwell, Masters, \& Eves, 2003; Poolton, Masters, \& Maxwell, 2005). It has been argued that analogy learning promotes efficient information processing by packaging movementspecific information in a single, meaningful unit, which reduces reliance on working memory (Liao \& Masters, 2001; Masters, 2000; Masters \& Liao, 2003). However, little evidence exists to support this claim.

Highly efficient cognitive processing is a trademark of expert performance. Hatfield and Hillman (2001) used the term psychomotor efficiency to describe refined allocation of neural resources, absence of effortful cognition (based on brain activity measures), and adaptive, efficient movements by experts. Generally, psychomotor efficiency may reflect either higher performance with reduced neural effort or at least no change in neural effort, or maintenance of performance using less neural effort. Psychomotor efficiency may therefore reflect less reliance on explicit, verbal information during preparation or performance of a movement, as seen in experts compared to novices (Hatfield \& Hillman, 2001), following practice (Landers et al., 1994) or between successful and unsuccessful executions (Hatfield \& Hillman, 2001; for a review see Hatfield, Haufler, Hung, \& Spalding, 2004). If analogies represent movement-specific information as a unit of information rather than as a collection of explicit rules, 
analogy instruction might reduce the amount of verbal-declarative information that is processed during motor preparation or performance and therefore increase psychomotor efficiency. Electroencephalography (EEG) provides an objective method by which to examine this possibility. EEG quantifies electrical signals on the scalp surface. The signals are measured at different frequencies, which reflect functionally different cognitive processes (Janelle et al., 2000; Ray \& Cole, 1985; see also Smith et al., 1999). Psychomotor processes involve medium-range communication between different brain areas, which manifests in signals of alpha-band frequency range (8-12 Hz, Crews \& Landers, 1993; Klimesch, 1999; Nunez \& Srinivasan, 2006). Alpha-wave activity measured on the scalp displays different cognitive functions depending on the exact scalp locations at which the electrical signals are measured. Verbal-cognitive activity, for example, is associated with alpha activity in the left temporal lobe, which underlies the T7 region in the International 10-20 system of electrode placement. Visuospatial processes are associated with activity in the right temporal lobe (T8 region) and motor planning is commonly associated with activity in the premotor cortex (frontal midline or Fz region, Kaufer \& Lewis, 1999).

Alpha band activity is, in general, inversely related to cortical activation, meaning that increased alpha power reflects decreased cerebral activation. Contrastingly to broadband alpha power, which represents general attention processes as well as task-specific processes, activity in the higher-frequency half of the bandwidth (high-alpha power) is more specifically related to long- and medium-range cortico-cortical communication (Crews \& Landers, 1993; Nunez \& Srinivasan, 2006; Janelle et al. 2000; Nunez et al. 1999). High-alpha power is representative of task-specific processes and interregional cross-communication (Smith et al. 1999; for a review see Klimesch, 1999). In various expertnovice comparison studies, expertise has been associated with higher alpha power, suggesting that experts are neurally more efficient than non-experts when programming and executing a task (Haufler, Spalding, Santa Maria, \& Hatfield, 2000). Studies have also found higher alpha power at frontal and central sites in successful compared to unsuccessful performance (e.g., Crews \& Landers, 1993; Hillman et al., 2000). Cooke et al. (2015) found less power in the high alpha band (10-12 Hz) during the pre-movement period in golf putting following errors (especially in experts), which they attributed 
to increased conscious activity associated with processing and correcting errors. For a review of recent research using psychophysiological measures in sport performance, see Cooke (2013). Neural coactivation between brain regions can provide further insight into psychomotor efficiency. Termed EEG coherence, this measure can tell us how much cross-communication occurs between two regions at a specified time. For example, coherence between signals measured at the left temporal and frontal midline regions indicates involvement of verbal brain areas during motor planning, and potentially is a marker of psychomotor efficiency (Hatfield \& Hillman, 2001).

If motor analogies convey complex motor skill information in the form of a simple concept or image, then an analogy instruction may lead to reduced information processing demands during motor planning of the specific skill. This increased psychomotor efficiency should be evident in reduced neural coactivation between the left temporal lobe and frontal midline during movement preparation (at least compared to explicit instructions or no instructions). Zhu, Poolton, Wilson, Maxwell, and Masters (2011b) showed that implicit motor learning (using an errorless approach during golf putting) resulted in less neural coactivation between the left temporal lobe (T3) ${ }^{1}$ and the frontal midline (Fz) than explicit motor learning, which supports this conclusion (see also continuous tracking; Zhu, Poolton, Wilson, Hu, et al., 2011a). Additionally, increased left-temporal high-alpha power should reflect economy of task-specific cognitive processes brought about by analogy learning. To date, no research has been conducted involving EEG alpha power as a measure of psychomotor efficiency in an implicit learning scenario, nor has any research generalized EEG findings related to error-reduced (implicit) learning (Zhu et al., 2011a; Zhu et al., 2011b) to analogy learning.

We developed an analogy instruction for a push-pass in field hockey ("move the [hockey] stick as if you are sloshing a bucket of water over the floor”) and asked whether it promoted higher psychomotor efficiency than explicit instructions (provided by a qualified coach) or no instructions. Psychomotor

\footnotetext{
${ }^{1}$ In this paper, all labels are translated to the nomenclature suggested in the American Electroencephalographic Society's "guidelines for standard electrode position nomenclature” (American Electroencephalographic Society, 1994; Chatrian et al., 1985; Jurcak et al., 2007; Klem et al., 1999). The terms T7 and T8 are used for left and right temporal lobes, while Fz is used for the frontal midline. Odd numbers indicate left hemisphere, even numbers indicate right hemisphere locations.
} 
efficiency was quantified by measuring left-temporal high-alpha power and T7-Fz (left temporal-

2

frontal) coherence. Participants performed the push-pass as a single task and as a combined task (with concurrent decision-making), both before and after practice (24h delayed retention). Combined tasks reduce the capacity available for motor processing (e.g. Koedijker et al., 2011; Lam et al., 2009b; Liao \& Masters, 2001) and therefore may be more sensitive to changes in efficiency than regular (singletask) tests. Based on previous findings (e.g. Liao \& Masters, 2001), task-related knowledge (measured by verbal protocols) was expected to be significantly lower in the Analogy instructed group compared to the Explicit and No-Instruction groups, while the latter two groups were not expected to differ. EEG activity was assessed during a 4s-preparation period prior to performing the push-pass alone (single task). We expected to find a greater increase in high-alpha power in the left temporal lobe (T7) following analogy instruction and a greater decrease in T7-Fz coherence, reflecting higher psychomotor efficiency. Alpha power at the right temporal lobe (T8) and coherence between T8 and frontal midline was also assessed, but no differences were expected. We expected greater psychomotor efficiency in people who were taught by analogy to be reflected by better push-pass performance in the combined task compared to people who were instructed with explicit rules or no instructions.

\section{Method}

\section{Participants}

Forty-eight novice hockey players (Mean age $=22.21, \mathrm{SD}=6.16$ ) with normal or corrected-to-normal vision and no movement impairments participated in the study. Participants were recruited from the University of Waikato student population, from classes and by word of mouth. Participants were incentivized to participate with cafeteria vouchers (value NZD10). Participants with more than 20 hours of experience were excluded from the study. Ethical permission for the study was received from the Faculty Ethical Committee of the institution. Participants all provided informed consent. 
2 Standard field hockey sticks (92.7 $\mathrm{cm}$ length) were used on an artificial grass surface. Standard

Wilson ${ }^{\circledR}$ tennis balls replaced hockey balls. Passes were directed toward a red semi-circle positioned on the wall at ground level, which was surrounded by 13 concentric semi-circles at $10 \mathrm{~cm}$ intervals (see Figure 1, left). The distance from the starting position to the target was $340 \mathrm{~cm}$. A Texas Instruments DLP projector was used to project images onto the blank laboratory wall. Performance (accuracy) was measured as distance from the target center and was obtained by manual analysis of video footage from a Sony RX10pi camera focused on the target.

\section{Electroencephalography}

EEG equipment included a wireless EEG / tDCS transmitter, a set of 4 measuring and 2 reference electrodes, conducting gel and electrode contact stickers (Neuroelectrics Barcelona, SLU, ESP). The system was connected to a desktop computer and analyzed using Neurosurfer software by Neuroelectrics. EEG activity was recorded from 6 silver/silver chloride (Ag/AgCl) electrodes on the scalp positioned using different sized neoprene caps with pre-defined holes. Two reference electrodes were placed at the earlobe using a clip. Where the earlobe was too small to hold the clip, two adhesive electrodes were attached to the left mastoid. Caps and electrodes were adjusted carefully in line with the 10-20 system (Chatrian, Lettich, \& Nelson, 1985) and checked by two technicians before the start of the experiment. Measurement electrodes were positioned at the left and right temporal lobes (T7, T8), at the frontal midline (Fz) and at the parietal lobe (Pz, control) of the scalp. Signals were collected at a sampling rate of $500 \mathrm{~Hz}$ with 24-bit resolution and 0 to $125 \mathrm{~Hz}$ bandwidth.

Prior to commencing the task, an appropriate level of impedance was achieved by adjusting electrode positions, the participant's hair and the amount of electrolyte gel. While an upper limit of $15 \mathrm{kOhm}$ was used for inclusion, impedance levels were generally below $5 \mathrm{kOhm}$. Potential issues associated with using repeated measures on two different days were overcome by fully standardizing the procedures and by scheduling the two sessions exactly 24h apart (see Ring, Cooke, Kavussanu, McIntyre, \& 
1 Masters, 2015) ${ }^{2}$ The pre-session criteria required participants to (a) wash their hair on the

2 evening/morning before testing, and (b) not consume caffeine in the 2 hours preceding testing.

\section{Procedure}

4

5

6

7

8

9

\section{General procedure}

Participants were randomly allocated to an analogy instruction group (AG; $n=17$ ), an explicit instruction group (EG; $\mathrm{n}=14$ ) or a no instruction group (NIG; $\mathrm{n}=17$ ), using a random number generator. They were asked to attend the laboratory on two separate days at the same time. On both days, participants were fitted with a Neuroelectrics cap and EEG electrodes were attached. Participants performed a hockey push-pass task in a pretest (day 1) and a retention test (day 2). Prior to completing the pretest, participants were shown two animations that illustrated the requirements of the push-pass; however, participants were not instructed how to move. A push-pass is a shot in which the hockey stick guides the ball towards a target without use of a backswing. In our experiment, it was required that the ball rolled along the ground. An animation that was described as the desired outcome showed a square (representing the hockey stick) and an adjacent circle (representing the ball) moving from the left to the right side of the screen. A second animation that was described as a negative outcome showed a circle resting in the middle of the screen and a square approaching it, "hitting” it towards the right side of the screen. In both the pretest and the retention test, a single task was performed (20 trials), followed by a combined task (20 trials). The single task required participants to pass the ball as accurately as possible to the target.

For the combined task, 20 images of hockey players $(n=3)$ standing in different positions were projected onto the opposing wall. Two players were wearing black shirts and one was wearing an orange shirt. Arrangements of the players varied, with one or two players in the foreground (85\% of life size) and the others in the background (70\% of life size, see Figure 1, right). Participants were informed that

\footnotetext{
${ }^{2}$ Baseline EEG data was collected on both days prior to commencing the experiment (120 seconds seated and motionless), in order to determine whether high-alpha power was comparable between day 1 and day 2 . Correlations were evident for T7 $(r=.646, \mathrm{p}<.001)$, T8 $(\mathrm{r}=.464, \mathrm{p}<.005)$ and $\mathrm{Fz}(\mathrm{r}=.358, \mathrm{p}<.05)$.
} 
they were a member of the black team and were to push-pass the ball as quickly and as accurately as possible towards the hockey stick (also 70 or $85 \%$ of life size, i.e. around $88 \mathrm{~mm}$ hook diameter) of the player who was in the best position to receive the ball. Presentation of each image was preceded by a brief countdown on a blank background.

At the end of the retention test, participants were required to complete verbal protocols in which they reported any rules or self-instructions that they recalled consciously using to control their movements. This approach was used as a manipulation check for the analogy instruction and to evaluate whether the No Instruction group had indeed acquired a similar amount of declarative knowledge to the Explicit group, which would be expected based on hypothesis testing.

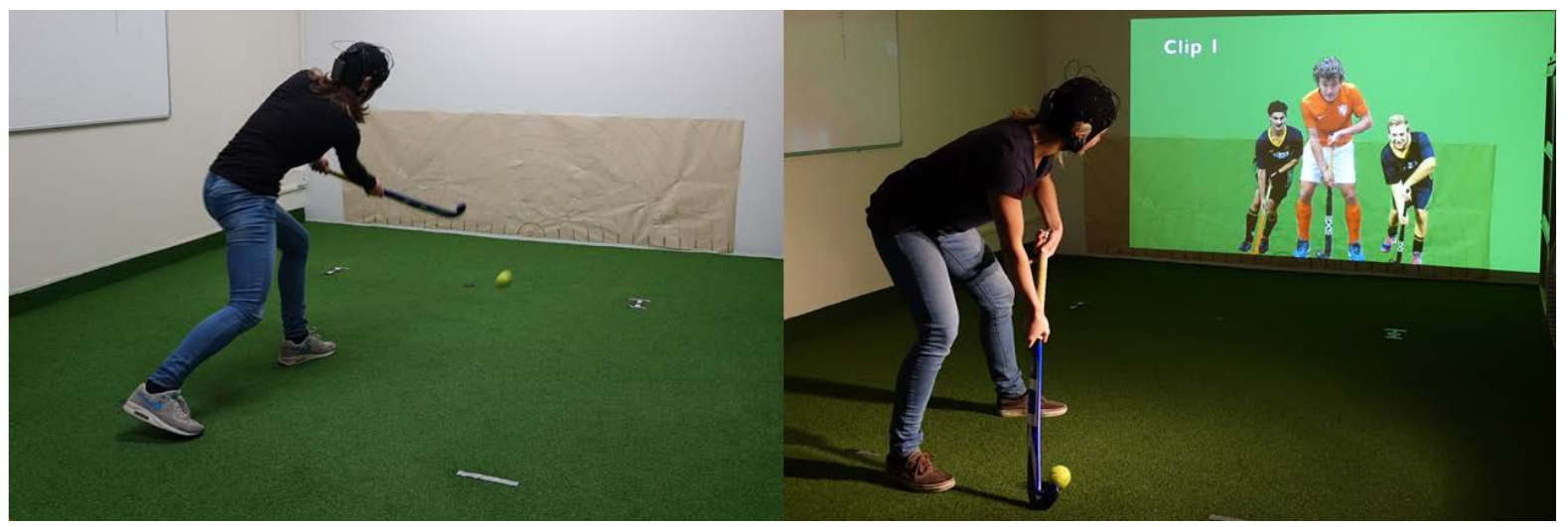

Figure 1. Execution of a push-pass in the single task (left) and combined task condition (right).

\section{Instruction and practice}

Between the pretest and retention test, participants were provided with the analogy instruction (AG), written instructions about how to execute a push-pass (EG), or no instructions (NIG) (see Table 1 for the exact instructions). They then practiced the push-pass by performing 4 blocks of 32 trials each. For each trial, they were required to pass the ball to a virtual teammate (75\% of life size) who moved from right to the left on the screen, and vice versa (random order). Participants were instructed that they should time the pass so that the ball would accurately hit the strike zone of their teammate's hockey stick. The strike zone was indicated by a yellow circle $(11 \mathrm{~cm}$ diam) bounded by a blue circle $(18 \mathrm{~cm}$ diam). An opponent/defender, played by one of the researchers, was positioned $1.3 \mathrm{~m}$ from the screen 
either directly in front of the participant or $90 \mathrm{~cm}$ to the right or left, respectively. The participant was instructed to pass the ball so that the opponent/defender could not intercept it. Before starting each block of trials, participants were asked to repeat the specific instructions that they had received, in order to ensure that they remembered them and used them consciously during the block. Performance was recorded and feedback (knowledge of results) was provided to participants at the end of each block in order to maintain motivation. ${ }^{3}$

Previous research has shown that analogies need to be meaningful and linked to personal experience in order to be of benefit during learning (e.g., Gentner, 1983; Poolton et al, 2007). Therefore, preceding the analogy instruction, participants in the AG performed a familiarization task designed to provide them with personal experience of the analogy concept. EG and NIG participants performed a control task of the same duration with the same materials ${ }^{4}$. Participants in the AG were provided a bucket of water and were instructed to knock over as many cones as possible (lined up on the floor in a single file) by "sloshing" the water underneath a bar raised $15 \mathrm{~cm}$ from the ground. The task, which was repeated twice, was designed to familiarize participants with the concept represented by the analogy (move the stick as if you are sloshing a bucket of water over the floor). Discussions with an experienced hockey player and an international coach suggested that the analogy (and the familiarization task) appropriately captured the concept of a push-pass (see Figure 2).

The control task for the EG and NIG participants was to scoop water from a full bucket into an empty one using a cup. The EEG cap worn by each participant was protected from water by a shower cap and the tasks were performed in an outdoor area next to the laboratory. After the familiarisation task, participants were provided with the analogy instruction (AG), written instructions about how to execute a push-pass (EG), or no instructions (NIG, see Table 1).

\footnotetext{
${ }^{3}$ The practice task was designed to be realistic and ecologically valid, but for practical reasons, and to maximize measurement accuracy, the pretest and retention test were conducted in a "laboratory style" manner. This mismatch in task specificity was held consistent across groups and was therefore not expected to lead to confounding results.

${ }^{4}$ We acknowledge that the experience of sloshing a bucket of water over the floor might have benefitted the Analogy group. However, this potential benefit was deemed to be minimal.
} 


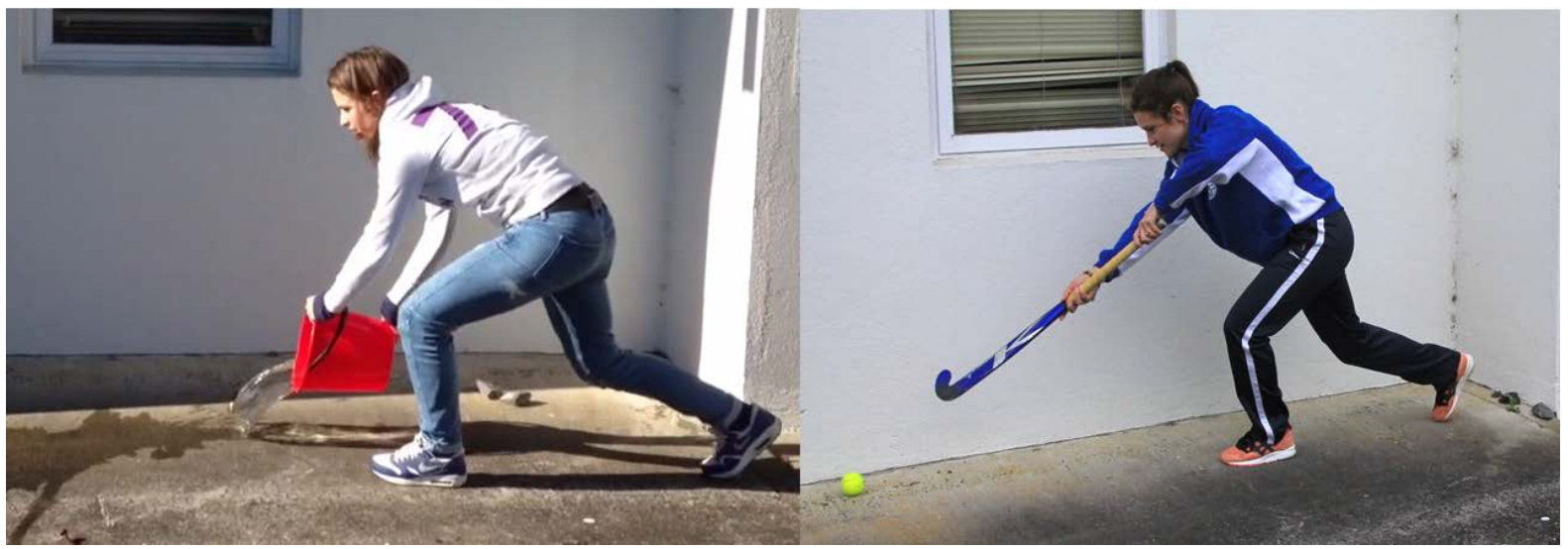

2 Figure 2. Performance of the familiarization task (left) vs. performance of a hockey push-pass (right).

3 Table 1. Push-pass instructions for each group

\begin{tabular}{ll}
\hline Group & Instructions \\
\hline Analogy & Move the [hockey] stick as if you are sloshing a bucket of water over the \\
& floor.
\end{tabular}

Explicit

Hold the stick with a wide grip

The ball should be in front of the right foot

Make a side step with the left foot

Pull the ball past the left foot

Keep contact with the ball while pushing

Finish by pointing at the target with the stick

No-instruction

Try to push the ball to the target as exactly as possible.

4

5

6 


\section{Dependent variables and data analysis}

2

\section{Dependent variables}

Measurements for both performance variables were made using video recordings collected at normal speed (30 fps) and played frame-by-frame in order to determine the time when the ball contacted the wall. Single task performance was represented by mean contact distance from the target during the single task test (20 trials). Combined task performance was represented by mean contact distance from the target chosen by the participant (20 trials). For both tasks, we were mostly interested in improvement due to the intervention; therefore we investigated performance change between the pretest and the retention test, calculated by subtracting mean distance at pretest from mean distance at retention ${ }^{5}$. A positive value represents worse performance, whereas a negative value represents improved performance. Verbal protocols were evaluated by counting each rule or self-instruction that concerned a separate aspect of the movement.

\section{Data Reduction}

EEG values were only recorded during the single task, because pilot work showed that the combined task required participants to scan a large area of the screen in front of them, which created EEG artefacts as a consequence of head movements. No baseline subtraction was performed. Raw EEG signals were bandpass filtered at 1-30Hz. Signals were then resampled at $256 \mathrm{~Hz}$. Data from the pretest and retention test were processed as 4 sec segments prior to movement initiation (which was recorded on the EEG timetrace during the experiment using manual markers). Blinks and eye movements are characterized by high potentials (Boudet, Peyrodie, Gallois, \& Vasseur, 2006). Eye artifacts were excluded by an extreme measures approach. Epochs (0.25s length) within each $4 \mathrm{~s}$ segment that contained signals above $60 \mu V^{2}$ were discarded. An average of $174.39(\mathrm{SD}=69.14)$ epochs per participant in the pretest and 167.35 $(\mathrm{SD}=67.14)$ epochs per participant in the retention test were retained. A Fast Fourier Transform with a Hamming window taper and 50\% overlap with a resolution of $0.49 \mathrm{~Hz}$, was applied to each $4 \mathrm{~s}$

\footnotetext{
${ }^{5}$ A one-way ANOVA revealed no significant differences between groups at pretest during the single task $(\mathrm{F}(2,45)$ $=1.417, p=.253)$ or the combined task $(\mathrm{F}(2,45)=1.778, p=.181)$.
} 
segment. EEG high-alpha $(10-12 \mathrm{~Hz})$ power was calculated for the 4 sec segment preceding each trial (e.g., Deeny et al., 2003). Manual manipulation of the trigger may have slightly compromised the validity of the power and coherence measures because of experimenter error, but considerable care was taken to avoid this. The EEG analyses in this study focused on the high-alpha (10-12 Hz) band. Activity in this bandwidth is representative of task-specific attention processes (e.g., Klimesch, 1999). Matlab scripts (MATLAB Release 2016b, The MathWorks, Inc., Natick, MA - US) were used to calculate EEG outputs. The processing and analysis steps described above were implemented with the EEGLAB toolbox (Delorme \& Makeig, 2004). EEG coherence was calculated using a formula based on Equation (1), in which Pxx and Pyy represent the power spectral density of signals at location $\mathrm{x}$ and $\mathrm{y}$, respectively, and Pxy represents the cross power spectral density of x and y (e.g., Zhu, Poolton, Wilson, Maxwell, et al., 2011). The measure was not $\mathrm{z}$ transformed.

$$
C_{x y}(f)=\frac{\left|P_{x y}(f)\right|^{2}}{P_{x x}(f) P_{y y}(f)}
$$

\section{Statistical analyses}

In order to test our a priori predictions concerning high-alpha power at T7, and T7-Fz coherence, as well as combined task performance, we conducted orthogonal contrasts. The NIG was expected to learn by discovery and thus to show the same behaviour as the EG. Orthogonal contrasts were therefore used to compare the AG against the EG and NIG in the first instance, and the EG against the NIG in the second instance. Based on unequal variances in high-alpha power at T7 variable, Bonferroni correction was used in this instance. We did not make clear predictions regarding changes in T8-Fz coherence, T8 or Fz high-alpha power, so mixed ANOVA with repeated measures was conducted to investigate alpha power at $\mathrm{Fz}$ and at $\mathrm{T} 8$ during the pretest and retention test and 3-way (between groups) univariate ANOVA was conducted on T8-Fz coherence. Mixed ANOVA with repeated measures on the test factor was used to investigate pretest and retention test single task performance in the different instruction groups. Orthogonal contrasts were also used to compare the number of rules reported in each group, 
1 since AG was expected to differ from the EG and NIG groups, which were not expected to differ.

2 Statistical significance was set at $\mathrm{p}<.05$ for all primary analyses.

[Please refer to Appendix for Table 2]

\section{Verbal protocols}

\section{Results}

Table 2 shows mean values during the pretest and retention test and change scores in performance and EEG power as well as coherence between the pretest and retention tests, for all groups collapsed and separately.

Orthogonal contrasts revealed a significant difference in movement-related knowledge (number of rules) between the analogy group $(\mathrm{M}=2.235$ / $\mathrm{SD}=1.393)$ compared with the explicit $(\mathrm{M}=3.786$ / $\mathrm{SD}=1.929)$ and no-instruction groups $(\mathrm{M}=3.412 / \mathrm{SD}=2.181 ; \mathrm{t}(45)=-2.426$, contrast $=-2.727, p=$ .019), but no significant difference between the explicit and the no-instruction group $(\mathrm{t}(45)=.557, p=$ $.580)$.

\section{EEG power and coherence}

EEG high-alpha power at T7, measured during the single task, was analysed using orthogonal contrasts. Change in EEG high-alpha power at T7 was significantly different in the AG compared to the EG and NIG combined $(\mathrm{t}(21.490)=-2.131$, contrast $=-2.172, p=.045)$. No difference was evident between EG and NIG $(\mathrm{t}(22.508)=.562 . p=.580)$. Figure 3 illustrates the mean change in high-alpha power for each group.

EEG coherence between the T7 and Fz regions, measured during the single task, was also analysed using orthogonal contrasts. Change in EEG coherence was not significantly different in AG compared to the EG and NIG combined ( $\mathrm{t}(45)=-.073, p=.942)$, nor was there a difference between the EG and NIG $(\mathrm{t}(45)=.795, p=.431)$. 
A 2 (test; pre, retention) x 3 (group; Analogy, Explicit, No Instruction) mixed ANOVA with repeated measures on the test factor was conducted to investigate T8-Fz coherence. No significant effect of test $\left(F(1,45)=1.528, p=.223\right.$, partial $\left.\eta^{2}=.033\right)$ or group $\left(F(2,45)=0.375, p=.689\right.$, partial $\left.\eta^{2}=.016\right)$ was found, and no significant interaction was evident $\left(\mathrm{F}(2,45)=.016, p=.984\right.$, partial $\left.\eta^{2}=.001\right)$.

A 2 (test; pre, retention) x 2 (location; T8, Fz) x 3 (group; Analogy, Explicit, No Instruction) mixed ANOVA with repeated measures on the test factor was conducted to investigate alpha power at $\mathrm{Fz}$ and at T8. No significant effect of test $\left(F(1,45)=3.741, p=.059\right.$, partial $\left.\eta^{2}=0.077\right)$, or group $(F(2,45)=$ $0.617, p=.544$, partial $\left.\eta^{2}=0.027\right)$ was found. A significant main effect was found for location $(F(1,45)$ $=4.107, p=.049$, partial $\left.\eta^{2}=0.084\right)$. Significant interactions were not evident between test $\mathrm{x}$ group $\left(F(2,45)=1.887, p=.163\right.$, partial $\left.\eta^{2}=.077\right)$ or location $\mathrm{x}$ group $\left(F(2,45)=0.820, p=.447\right.$, partial $\eta^{2}$ $=0.035)$, but a significant interaction was found between test $\mathrm{x}$ location $(F(1,45)=5.863, p=.020$, partial $\left.\eta^{2}=.115\right)$

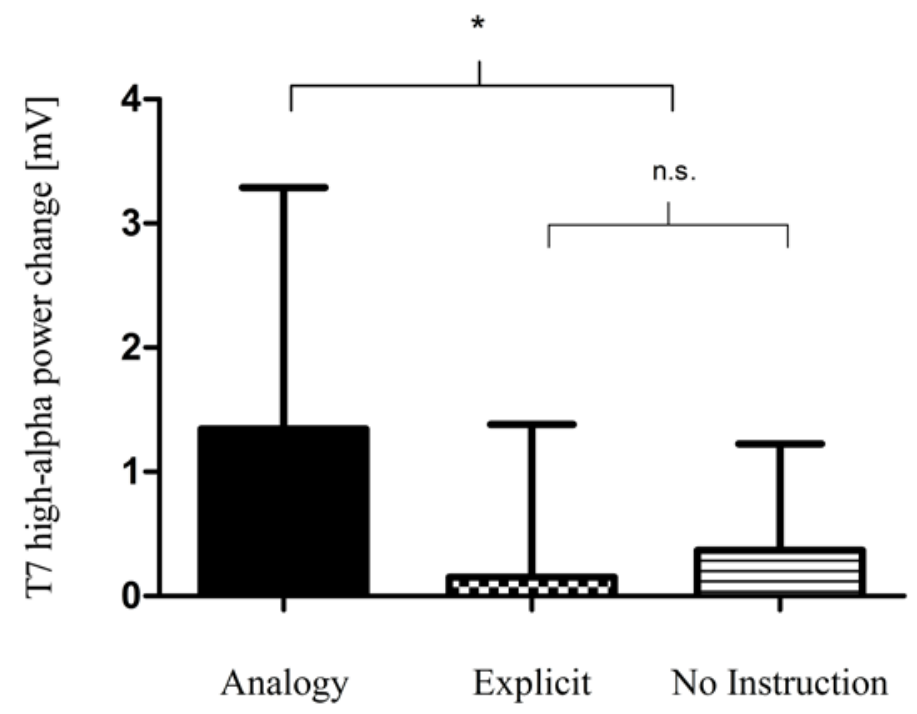

Group

Figure 3. Change $\left(\mathrm{mV}^{2}\right)$ in high-alpha power at $\mathrm{T} 7$ between the pretest and retention test. A higher value indicates increased power (but reduced activation). * indicates a significant difference between groups. 
2

3

4

5

$6 \eta^{2}=.053$ ) were found.

7

8

9 10 $1.025, p=.311$ ). Figure 4 shows combined task performance change for each group.

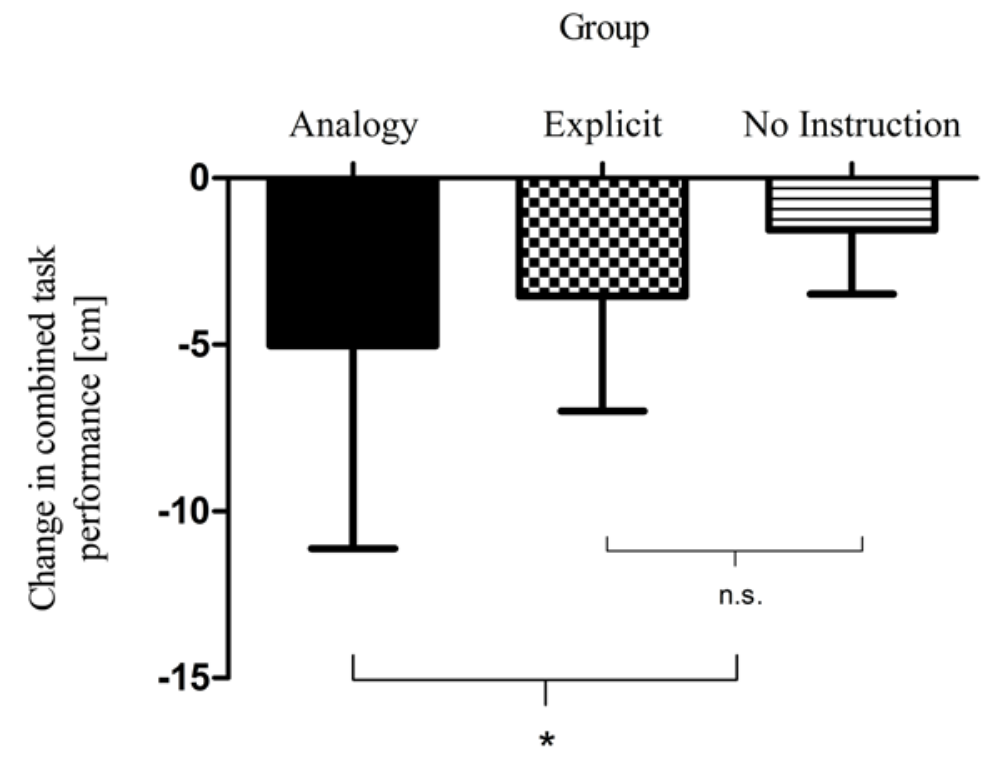

Figure 4. Change $(\mathrm{cm})$ in combined task performance between the pretest and the retention test. A groups. 
1 We investigated whether using an analogy instruction leads to increased psychomotor efficiency 2 compared to explicit instructions or no instructions (discovery). Previous research has shown that

analogy learning limits the accrual of task-related, verbalisable knowledge compared to uninstructed discovery learning or explicit instructions (e.g., Liao \& Masters, 2001). Verbal protocols collected as a manipulation check following the learning phase in our study showed that movement-related knowledge (number of rules) differed significantly between the analogy group compared with the explicit and noinstruction groups, but not between the explicit and the no-instruction group.

While all participants improved in single task performance following practice, improvement did not differ between instruction groups. This meets expectations, considering that changes in efficiency due to analogy instructions were expected to happen on a cognitive rather than a motor level, and thus were less likely to impact motor performance in the single task to the same extent as the combined task. The analogy group showed greater improvement in combined task performance compared to the explicit and no instruction groups. This finding is consistent with previous research on analogy learning and confirms the validity of the hockey push pass analogy developed for this study. We speculate that this finding might also provide some support for our hypothesis that analogy instructions promote efficiency in verbal-cognitive processing related to motor planning, as it suggests that motor planning following analogy instruction was successful even when cognitive resources were limited by the secondary task (choosing the target). However, a limitation of the current study is that EEG data was not collected during the combined task. If we had data on EEG high-alpha power in the left temporal region during the combined task, which is a useful index of verbal-cognitive processing, we would be able to conclude more strongly.

With respect to our EEG measures, differences between the instruction groups were evident in verbal-cognitive brain regions (left temporal lobe), but not in motor planning or visuo-spatial regions, with an increase in EEG high-alpha power evident in the left temporal lobe for the analogy group compared to the explicit and no instruction groups. This finding provides support for our premise that cognitive efficiency may be promoted by analogy instructions relative to explicit instructions or no instructions. Increased high alpha power at the left temporal lobe may imply a reduction in verbal- 
cognitive processing. ${ }^{6}$ Efficiency is defined as better performance related to less effort. Since the analogy group displayed performance improvements during the single task that were similar to the other groups, and also displayed increased high-alpha power at the left temporal lobe, it is possible that the analogy group exhibited greater efficiency in verbal-cognitive processing relative to the other two groups. EEG high-alpha power in the Fz and T8 regions did not differ between the groups, which is consistent with our expectations; activation in these areas was not expected to become more efficient due to the analogy instruction. High-alpha power at Fz represents motor planning processes, which did not seem to be affected by analogy instructions. Since no differences were found in motor performance (single task), these results fit well with the neural data. Regular motor performance, as well as neural processes related to motor planning, did not improve due to the analogy instruction.

EEG coherence between the left temporal lobe and the frontal midline (T7 and Fz) did not show the expected difference between groups, thus the hypothesis that an analogy instruction would affect this measure of psychomotor efficiency was not supported. This finding may indicate that analogies affect efficiency of verbal-cognitive processes but not verbal-cognitive motor control. Since T7-Fz coherence reflects verbal-motor cross-communication, including motor planning processes (Deeny, Hillman, Janelle, \& Hatfield, 2003), the measure is likely linked to efficiency in motor planning and verbal processing combined rather than verbal processing alone. This notion is supported by work of Zhu et al. (2011), who showed a connection between T7-Fz coherence and motor performance in a single task as well as dual task condition. In their experiment, participants in an implicit (error-reduced) treatment condition exhibited lower T7-Fz coherence compared to participants in an explicit (errorful) treatment condition. This decrease in T7-Fz coherence was not only connected to reduced mental load, reflected by superior dual-task performance, but also to performance benefits at a purely motor level, as shown by improved single task performance. Therefore, coherence may not represent efficiency of verbalcognitive processes alone but rather efficiency of psychomotor processes (see Hatfield and Hillman, 2001). Although learning by analogy has been linked to implicit motor learning, the mechanism that underpins analogy instruction may be different from error-reduced learning and may only affect

\footnotetext{
${ }^{6}$ We acknowledge that EEG indices provide only an indirect measure of verbal processing.
} 
1 efficiency of the verbal-cognitive aspect of information processing. The notion that learning by analogy

2 affects cognitive but not motor processes finds some support from work by Lam et al. (2009b), which

failed to find significant differences in kinematic outcomes between analogy and explicit learners in a basketball shooting task. The authors concluded that analogy learning may not cause qualitative differences in the way that a motor task is performed.

The fact that analogy instructions may result in more efficient verbal-cognitive processing explains why performance differences in dual-task tests, but not single-task tests, have been found in previous analogy learning research (Koedijker et al., 2011; Lam et al., 2009b; Liao \& Masters, 2001). To date, there has been little evidence that dual-task performance benefits can be attributed to increased efficiency. However, the present study provides objective neural evidence that this is the case. The mechanism by which analogy instructions render cognitive processes more efficient remains unclear, however.

A tentative mechanism underpinning the effects of analogy learning uses the concept of chunking (Masters, 2000; Masters \& Liao, 2003), during which information becomes organized into higher order structures - many small ‘bits’ of information are collapsed into fewer larger chunks. Although fewer chunks are processed, they contain the relevant information, meaning that information can be processed with relatively less cognitive effort; processing becomes more efficient (Newell \& Rosenbloom, 1980). This chunking process is a part of the learning process. Masters and Liao (2003) suggested that during motor learning, slow natural chunking processes can be accelerated by using analogy. The analogy has been described as an "all encompassing, biomechanical metaphor” (Koedijker et al., 2011; Liao \& Masters, 2001; Masters, 2000). Since all necessary information for the fulfillment of the task is condensed into the analogy, it represents a higher level of organization among the rules for the movement rather than explaining the task step-by-step (Gentner, 1983). Analogy instructions may thus render information processing more efficient by chunking movement relevant information into a single unit or concept (Masters, 2000). However, this theory has not been backed by strong empirical support to date. Gathering sufficient evidence to either corroborate or discard such a chunking hypothesis of implicit motor learning is an important next research step to determine the mechanism of analogy 
1 learning.

\section{Conclusion}

3 In this experiment, we observed that people who were instructed by analogy improved more distinctly 4 at a combined task compared to people who practised using explicit instructions or no instructions. 5 Increased EEG high-alpha power at verbal processing regions of the brain during performance 6 suggested that analogy instructions may have resulted in more efficient verbal-cognitive processing 7 compared to learning by explicit instructions. Brain regions related to purely motor aspects of the 8 movement did not display increased efficiency. These findings lead us to contend that benefits of 9 analogy instructions may reflect improved efficiency in the processing of verbal-cognitive information, 10 but not in motor planning. In future work, we plan to use dual-task or combined task settings that do not require head movements, during which EEG variables can be collected. 
2 Berry, D. C., \& Broadbent, D. E. (1984). On the relationship between task performance and associated verbalizable knowledge. The Quarterly Journal of Experimental Psychology Section A: Human Experimental Psychology, 36(2), 209-231.

Boudet, S., Peyrodie, L., Gallois, P., \& Vasseur, C. (2006). A global approach for automatic artifact removal for standard EEG record. In Annual International Conference of the IEEE Engineering in Medicine and Biology - Proceedings (pp. 5719-5722). IEEE. http://doi.org/10.1109/IEMBS.2006.259237

Chatrian, G. E., Lettich, E., \& Nelson, P. L. (1985). Ten percent electrode system for topographic studies of spontaneous and evoked EEG activities. American Journal of EEG Technology, 25(2), 83-92.

Cooke, A. (2013). Readying the head and steadying the heart: a review of cortical and cardiac studies of preparation for action in sport. International Review of Sport and Exercise Psychology, 6(1), 117. http://doi.org/10.1080/1750984X.2012.724438

Cooke, A., Gallicchio, G., Kavussanu, M., Willoughby, A., McIntyre, D., \& Ring, C. (2015). Premovement high-alpha power is modulated by previous movement errors: Indirect evidence to endorse high-alpha power as a marker of resource allocation during motor programming. Psychophysiology, 52(7), 977-981. http://doi.org/10.1111/psyp.12414

Crews, D. J., \& Landers, D. M. (1993). Electroencephalographic measures of attentional patterns prior to the golf putt. Medicine \& Science in Sports \& Exercise, 25(1), 116-26.

Deeny, S. P., Hillman, C. H., Janelle, C. M., \& Hatfield, B. D. (2003). Cortico-cortical communication and superior performance in skilled marksmen: An EEG coherence analysis. Journal of Sport \& Exercise Psychology, 25(2), 188-204.

Delorme, A., \& Makeig, S. (2004). EEGLAB: An open source toolbox for analysis of single-trial EEG dynamics including independent component analysis. Journal of Neuroscience Methods, 
2

Donnelly, C. M., \& McDaniel, M. A. (1993). Use of analogy in learning scientific concepts. Journal of Experimental Psychology: Learning, Memory, and Cognition, 19(4), 975-87.

Gentner, D. (1983). Structure Mapping: A Theoretical Framework for Analogy. Cognitive Science, 7(2), 155-170. http://doi.org/10.1016/S0364-0213(83)80009-3

Hardy, L., Mullen, R., \& Jones, G. (1996). Knowledge and conscious control of motor actions under stress. British Journal of Psychology, 87(4), 621-36.

Hatfield, B. D., \& Hillman, C. H. (2001). The psychophysiology of sport: A mechanistic understanding of the psychology of superior performance. In R. Singer, H. A. Hausenblas, \& C. M. Janelle (Eds.), Handbook of Sport Psychology (2nd ed., pp. 243-259). New York: Wiley \& Sons.

Haufler, A. J., Spalding, T. W., Santa Maria, D. L., \& Hatfield, B. D. (2000). Neuro-cognitive activity during a self-paced visuospatial task: Comparative EEG profiles in marksmen and novice shooters. Biological Psychology, 53(2), 131-160. http://doi.org/10.1016/S0301-0511(00)00047-8

Hayes, N. A., \& Broadbent, D. E. (1988). Two modes of learning for interactive tasks. Cognition, 28(3), 249-276.

Hillman, C. H., Apparies, R. J., Janelle, C. M., \& Hatfield, B. D. (2000). An electrocortical comparison of executed and rejected shots in skilled marksmen. Biological Psychology, 52(1), 7183. http://doi.org/10.1016/S0301-0511(99)00021-6

Janelle, C. M., Hillman, C. H., Apparies, R. J., Murray, N. P., Meili, L., Fallon, E. A., \& Hatfield, B. D. (2000). Expertise differences in cortical activation and gaze behavior during rifle shooting. Journal of Sport and Exercise Psychology, 22(2), 167-182.

Kaufer, D. I., \& Lewis, D. A. (1999). Frontal lobe anatomy and cortical connectivity. In B. L. Miller \& J. L. Cummings (Eds.), The Human Frontal Lobes: Functions and Disorders (pp. 27-44). New York City, NY: Guilford press. 
1 Klimesch, W. (1999). EEG alpha and theta oscillations reflect cognitive and memory performance: a review and analysis. Brain Research Reviews, 29(2-3), 169-195. http://doi.org/10.1016/S01650173(98)00056-3

Koedijker, J. M., Oudejans, R. R. D., \& Beek, P. J. (2007). Explicit rules and direction of attention in learning and performing the table tennis forehand. International Journal of Sport Psychology, 38(2), 227-244.

Koedijker, J. M., Poolton, J. M., Maxwell, J. P., Oudejans, R. R. D., Beek, P. J., \& Masters, R. S. W. (2011). Attention and time constraints in perceptual-motor learning and performance: instruction, analogy, and skill level. Consciousness and Cognition, 20(2), 245-56.

Lam, W. K., Maxwell, J. P., \& Masters, R. S. W. (2009a). Analogy learning and the performance of motor skills under pressure. Journal of Sport and Exercise Psychology, 31(3), 337-357.

Lam, W. K., Maxwell, J. P., \& Masters, R. S. W. (2009b). Analogy versus explicit learning of a modified basketball shooting task: performance and kinematic outcomes. Journal of Sports Sciences, 27(2), 179-191. http://doi.org/10.1080/02640410802448764

Liao, C. M., \& Masters, R. S. W. (2001). Analogy learning: a means to implicit motor learning. Journal of Sports Sciences, 19(5), 307-19.

Masters, R. S. W. (1992). Knowledge, knerves and know-how: The role of explicit versus implicit knowledge in the breakdown of a complex motor skill under pressure. British Journal of Psychology, 3(83), 343-358.

Masters, R. S. W. (2000). Theoretical aspects of implicit learning in sport. International Journal of Sport Psychology, 31(4), 530-541.

Masters, R. S. W., \& Liao, C. M. (2003). Chunking as a characteristic of implicit motor learning. In XIth European Congress of Sport Psychology (pp. 137-138). Copenhagen, Denmark.

Masters, R. S. W., Poolton, J. M., \& Maxwell, J. P. (2008). Stable implicit motor processes despite aerobic locomotor fatigue. Consciousness and Cognition, 17(1), 335-338. 
http://doi.org/10.1016/j.concog.2007.03.009

Masters, R. S. W., Poolton, J. M., Maxwell, J. P., \& Raab, M. (2008). Implicit motor learning and complex decision making in time-constrained environments. Journal of Motor Behavior, 40(1), 71-9.

Maxwell, J. P., Masters, R. S. W., \& Eves, F. F. (2000). From novice to no know-how: A longitudinal study of implicit motor learning. Journal of Sports Sciences, 18(2), 111-120.

Maxwell, J. P., Masters, R. S. W., \& Eves, F. F. (2003). The role of working memory in motor learning and performance. Consciousness and Cognition, 12(3), 376-402.

Maxwell, J. P., Masters, R. S. W., Kerr, E., \& Weedon, E. (2001). The implicit benefit of learning without errors. The Quarterly Journal of Experimental Psychology, 54(4), 1049-1068.

Nunez, P. L., Silberstein, R. B., Shi, Z., Carpenter, M. R., Srinivasan, R., Tucker, D. M., ... Wijesinghe, R. S. (1999). EEG coherency II: experimental comparisons of multiple measures. Clinical Neurophysiology, 110(3), 469-486. http://doi.org/10.1016/S1388-2457(98)00043-1

Nunez, P. L., \& Srinivasan, R. (2006). A theoretical basis for standing and traveling brain waves measured with human EEG with implications for an integrated consciousness. Clinical Neurophysiology, 117(11), 2424-2435. http://doi.org/10.1016/j.clinph.2006.06.754

Poolton, J. M., Masters, R. S. W., \& Maxwell, J. P. (2005). The relationship between initial errorless learning conditions and subsequent performance. Human Movement Science, 24(3), 362-378.

Poolton, J. M., Masters, R. S. W., \& Maxwell, J. P. (2006). The influence of analogy learning on decision-making in table tennis: Evidence from behavioural data. Psychology of Sport and Exercise, 7(6), 677-688.

Poolton, J. M., Masters, R. S. W., \& Maxwell, J. P. (2007). Passing thoughts on the evolutionary stability of implicit motor behaviour: performance retention under physiological fatigue. Consciousness and Cognition, 16(2), 456-68.

Ray, W., \& Cole, H. (1985). EEG alpha activity reflects attentional demands, and beta activity reflects 
1 emotional and cognitive processes. Science, 228(4700), 750-752.

2 http://doi.org/10.1126/science.3992243

3 Ring, C., Cooke, A., Kavussanu, M., McIntyre, D., \& Masters, R. (2015). Investigating the efficacy of $4 \quad$ neurofeedback training for expediting expertise and excellence in sport. Psychology of Sport and Exercise, 16, 118-127. http://doi.org/10.1016/j.psychsport.2014.08.005

6 Schücker, L., Ebbing, L., \& Hagemann, N. (2010). Learning by analogies: Implications for performance and attentional processes under pressure. Human Movement, 11(2), 191-199.

8

Smith, M. E., McEvoy, L. K., \& Gevins, A., M. E. (1999). Neurophysiological indices of strategy development and skill acquisition. Cognitive Brain Research, 7(3), 389-404.

Van Duijn, T., Buszard, T., Hoskens, M. C. J., \& Masters, R. S. W. (2017). Discerning measures of conscious brain processes associated with superior early motor performance: Capacity, coactivation, and character. In M. R. Wilson, V. Walsh, \& B. Parkin (Eds.), Progress in Brain Research (Vol. 234, pp. 245-261). Amsterdam: Academic Press. http://doi.org/10.1016/S00652504(10)42003-6

Zhu, F. F., Poolton, J. M., Wilson, M. R., Hu, Y., Maxwell, J. P., \& Masters, R. S. W. (2011a). Implicit motor learning promotes neural efficiency during laparoscopy. Surgical Endoscopy, 25(9), $2950-2955$.

Zhu, F. F., Poolton, J. M., Wilson, M. R., Maxwell, J. P., \& Masters, R. S. W. (2011b). Neural coactivation as a yardstick of implicit motor learning and the propensity for conscious control of movement. Biological Psychology, 87(1), 66-73. 


\begin{tabular}{|c|c|c|c|c|c|c|c|c|}
\hline \multirow[t]{2}{*}{ Group } & \multirow[t]{2}{*}{ Test } & \multicolumn{3}{|c|}{ High-alpha power $\left[\mu \mathrm{V}^{2}\right]$} & \multicolumn{2}{|c|}{ High-alpha coherence (0-1) } & \multicolumn{2}{|c|}{ Performance (distance from target $[\mathrm{cm}]$} \\
\hline & & $\mathrm{T} 7$ & $\mathrm{~T} 8$ & $\mathrm{Fz}$ & $\mathrm{T} 7-\mathrm{Fz}$ & T8-Fz & Single & Combined \\
\hline \multirow[t]{3}{*}{ Analogy } & Pretest & $3.917(2.537)$ & $4.383(1.704)$ & $3.190(1.046)$ & $.466(0.135)$ & $.417(0.145)$ & $22.000(11.363)$ & $31.286(11.156)$ \\
\hline & Retention test & $5.263(3.060)$ & $4.929(2.578)$ & $3.727(1.547)$ & $.466(0.182)$ & $.439(0.180)$ & $15.757(5.840)$ & $20.164(6.065)$ \\
\hline & Change pre-ret & $1.346(1.941)$ & $.546(2.263)$ & $.537(1.491)$ & $-.0001(0.170)$ & $.021(0.188)$ & $-6.243(9.444)$ & $-11.123(8.558)$ \\
\hline \multirow[t]{3}{*}{ Explicit } & Pretest & $3.697(0.878)$ & 4.439 (1.818) & $3.058(1.025)$ & $.495(0091)$ & $.433(0.130)$ & $20.605(13.000)$ & 29.855 (13.443) \\
\hline & Retention test & $3.848(1.512)$ & $4.513(1.943)$ & $3.431(1.121)$ & $.468(0.153)$ & $.464(0.122)$ & $16.625(6.455)$ & $22.870(8.005)$ \\
\hline & Change pre-ret & $.151(1.232)$ & $.074(1.139)$ & $.373(1.158)$ & $-.027(0.148)$ & $.031(0.072)$ & $-3.980(8.702)$ & $-6.985(11.625)$ \\
\hline \multirow[t]{3}{*}{ No Instr. } & Pretest & 3.557 (1.319) & $4.562(1.256)$ & $3.031(0.790)$ & $.464(0.157)$ & $.451(0.158)$ & $16.175(6.510)$ & $24.643(7.313)$ \\
\hline & Retention test & $3.927(1.494)$ & $4.445(1.036)$ & $3.133(0.867)$ & $.483(0.102)$ & $.478(0.103)$ & $14.162(5.419)$ & $21.160(6.273)$ \\
\hline & Change pre-ret & $.370(0.856)$ & -.117 (1.269) & $.102(0.743)$ & $.020(0.163)$ & $.026(0.140)$ & $-2.013(5.418)$ & $-3.483(8.309)$ \\
\hline
\end{tabular}

3 
